\title{
Treatment of Hypothalamic Obesity with Dextroamphetamine: A Case Series
}

\author{
Christian Denzer $^{a} \quad$ Friederike Denzer ${ }^{a}$ Belinda S. Lennerz ${ }^{b}$ \\ Heike Vollbach $^{a}$ Robert H. Lustig ${ }^{c}$ Martin Wabitsch ${ }^{a}$ \\ aDivision of Pediatric Endocrinology and Diabetes, Department of Pediatrics and

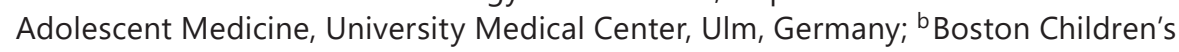 \\ Hospital, Boston, MA, USA; 'Division of Endocrinology, Department of Pediatrics, \\ University of California, San Francisco, CA, USA
}

\section{Keywords}

Hypothalamic obesity · Craniopharyngioma $\cdot$ Hyperphagia $\cdot$ Dextroamphetamine $\cdot$ Central nervous system stimulants

\begin{abstract}
Background: A limited number of published case reports suggest a positive effect of dextroamphetamine, an adrenergic agonist affecting both the central nervous system (CNS) and peripheral nervous system, on physical activity and weight in patients with hypothalamic obesity (intractable obesity following CNS insult). Here, we present our clinical experience with dextroamphetamine treatment for hypothalamic obesity. Methods: The clinical course of all patients started on dextroamphetamine treatment for severe hypothalamic obesity at our institution between 2010 and 2013 is reported. Dextroamphetamine administration was initiated at a single dose of $5 \mathrm{mg}$ per day and titrated to effect up to a dose of $20 \mathrm{mg} /$ day. BMI z-score velocity was calculated as change in BMI z-score over standardized intervals of 12 months. Parameters of treatment success and adverse events were assessed in a standardized fashion. Results: Seven patients (2 males; mean age 17.6 years [range 12.9-24.5]) underwent individual treatment attempts with dextroamphetamine between 2010 and 2013. The primary diagnoses were craniopharyngioma $(n=4)$, ganglioglioma WHO I $(n=1)$, astrocytoma $(n=$ $1)$, and neonatal meningitis $(n=1)$. Time from initial CNS insult to initiation of dextroamphetamine treatment averaged 5.2 years (range 2.4 months to 16.5 years). All patients demonstrated a steady increase in BMI z-score from the time of initial diagnosis until initiation of dextroamphetamine treatment. Mean baseline BMI z-score was $+3.17 \pm 0.93(+1.9$ to +4.4$)$.
\end{abstract}


Mean BMI z-score velocity decelerated to $-0.18 \pm 0.12$ per year during the first year of treatment and stabilized at $+0.05 \pm 0.32$ per year during the second year of treatment. No significant adverse events were reported. Conclusion: Dextroamphetamine treatment led to stabilization or reduction of BMI z-score in a cohort of 7 patients with hypothalamic obesity, with no adverse effects. Considering the projected increase in BMI z-score according to the natural course of the disease, these findings are promising and warrant further study.

(C) 2019 The Author(s)

Published by S. Karger AG, Basel

\section{Introduction}

Hypothalamic obesity is a complex neuroendocrine disorder caused by hypothalamic damage resulting in severe dysregulation of energy homeostasis [1]. Key centers for the integration of afferent and efferent homeostatic signals regulating body weight are located in the anterior (paraventricular nucleus), middle (arcuate nucleus, ventromedial nucleus), and posterior hypothalamus (dorsomedial nucleus, dorsal hypothalamic area). In the majority of cases, hypothalamic obesity is caused by damage of hypothalamic nuclei due to growth of sellar tumors with rostral extension, suprasellar tumors, neurosurgical procedures, cranial irradiation, head trauma, or inflammatory diseases [2, 3]. Hypothalamic obesity may also manifest in the context of multifaceted syndromal disorders (e.g., ROHHAD syndrome) and as a consequence of single gene mutations disrupting feeding pathways (e.g., LEP, LEPR) [2, 4].

In general, the clinical picture of hypothalamic obesity is characterized by rapid weight gain, extreme obesity, hyperphagia, decreased resting energy expenditure, and reduced physical activity. These key features of hypothalamic obesity have been most rigorously studied in craniopharyngioma patients [5]. Craniopharyngioma is the most common, nonglial intracranial tumor in childhood (incidence rate $0.5-2 / 1,000,000$ ), accounts for $1.2-4 \%$ of all brain tumors, and is presumably the most common cause of hypothalamic obesity. Altered eating behavior and extreme obesity develop in $40-50 \%$ of all craniopharyngioma patients. Although $12-19 \%$ of affected patients are already obese at diagnosis, the most rapid, uncontrollable weight gain usually occurs during the first 6-12 months following neurosurgical intervention [6]. Adding to the well-established characteristics of hypothalamic obesity, extremely obese craniopharyngioma patients typically suffer from panhypopituitarism and demonstrate significant hyperleptinemia due to anatomic leptin resistance, inadequate hyperinsulinemia for their degree of obesity, impaired incretin response, and reduced levels of blood catecholamines and urinary metabolites, and $\alpha$-MSH $[5,7,8]$.

Dextroamphetamine is an established treatment option for attention deficit and attention deficit hyperactivity disorders in childhood and adolescence. Decreased growth velocity compared to controls is noted, and in up to $76 \%$ of treated patients, moderate weight loss is observed [9]. Historically, amphetamines played a major role in pharmacologic obesity treatment during the 1940s and 1950s. Safety concerns regarding the addictive potential, and also postmarketing surveillance data linking certain amphetamines (dexfenfluramine, fenfluramine) with an increased risk for valvular heart disease and pulmonary hypertension, led to loss of approval and market withdrawals in many countries worldwide, dramatically cutting back the formerly widespread use of amphetamines for weight management (reviewed in [10]).

Two small, previously published case series (cumulative $n=17$ ) suggest a positive effect of dextroamphetamine treatment on body weight in patients with hypothalamic obesity [11, $12]$, reporting either weight stabilization or even moderate weight loss over the course of 7-45 months of dextroamphetamine treatment. Based on these observations, patients with 
Denzer et al.: Dextroamphetamine Treatment for Hypothalamic Obesity

hypothalamic obesity presenting to our endocrine outpatient clinic have been offered the use of dextroamphetamine as individual treatment attempts since the year 2010. Here, we share our clinical experience with dextroamphetamine treatment for hypothalamic obesity, highlight its potential role as an adjunct to the limited arsenal of treatment options, and underscore the need for clinical trials on stimulant treatment for hypothalamic obesity.

\section{Research Design and Methods}

\section{Study Population}

This retrospective case series comprises all patients who presented to our department with hypothalamic obesity (BMI >90th percentile according to German reference values $[13,14]$ ) following various central nervous system (CNS) insults between 2010 and 2013, and who consented to off-label dextroamphetamine treatment as individual treatment attempt for their otherwise intractable obesity, uncontrolled weight gain, low activity level, and daytime sleepiness. Patients and parents were informed comprehensively about the off-label use, effects, and potential side effects of dextroamphetamine by trained pediatricians. Consent was formally documented in patient files. Approval by the ethics committee was not required for individual treatment attempts. All patients underwent baseline evaluations to exclude contraindications for treatment with adrenergic stimulants (seizure disorders, depression, mood disorders, cardiovascular disease, hypertension, and arrhythmia). This included a neurologic examination by a pediatric neurologist, an electroencephalogram, a psychologic evaluation by a clinical psychologist, an in-depth cardiologic evaluation by a pediatric cardiologist including echocardiography, electrocardiogram, and blood pressure measurements. Dextroamphetamine administration was initiated at a single dose of $5 \mathrm{mg}$ per day orally and titrated to effect up to a dose of $20 \mathrm{mg}$ /day divided in 2-3 single doses (Table 2). For documentation of potential side effects, treating physicians obtained a specific medical history at each visit to the outpatient unit using standardized questions covering cardiovascular (palpitations, tachycardia), CNS (aggressiveness, dysphoria, tics, headache, restlessness, insomnia), dermatologic (alopecia, urticaria), gastrointestinal (constipation, diarrhea), neuromuscular (dyskinesia, tremor), and ophthalmic (blurred vision) adverse reactions.

\section{Anthropometric Measurements}

Body weight and height were determined to the nearest $0.1 \mathrm{~kg}$ and $0.1 \mathrm{~cm}$ using calibrated scales at treatment initiation and at each follow-up visit scheduled in 3-monthly intervals. BMI z-scores were calculated using the LMS method [15] based on German references [13, 14]. As follow-up data was derived from clinical evaluation, follow-up intervals were not consistent, and often less frequent than the desired intervals of 3 months between visits. To adjust for different follow-up intervals, annualized BMI z-score velocity was calculated. BMI z-score velocity represents the change of BMI z-score over a standardized interval of 12 months in analogy to the calculation of height or weight velocity [16]. Intervals between 9 and 15 months between individual BMI z-score measurements were considered acceptable for calculation of BMI z-score velocity. A retrospective chart review was conducted to establish BMI z-score velocity prior to treatment initiation.

\section{Statistical Procedures}

Descriptive data analysis was performed using the Aabel software package version 9.2 (Gigawiz Ltd. Co., Tulsa, OK, USA). In tables, data are presented as mean and standard deviation. Bar charts report means and respective standard deviations indicated by error bars. Box plots are composed of three horizontal lines that display the 10th, 50th, and 90th percentile of a variable. Whiskers are extended to extreme data points.

\section{Results}

In this case series, we report the clinical course of 7 patients $(2$ males $/ 5$ females, mean age 17.6 years, range 12.9-24.5 years). The primary diagnosis was craniopharyngioma in 4 patients, and ganglioneuroma, astrocytoma, and neonatal meningitis in the remaining 3 , respectively. Average time from initial CNS insult to initiation of dextroamphetamine treatment was 5.2 years (median 3.5 years, range 2.4 months to 16.5 years). 
Denzer et al.: Dextroamphetamine Treatment for Hypothalamic Obesity

\section{Case Vignettes}

Patient 1

Patient 1 initially presented to the pediatric endocrinology outpatient unit for evaluation of short stature at the age of 14 years and 6 months. Following normal longitudinal growth along the 10 th to 25 th percentile, height had dropped below the 3rd percentile (z-score -3.92) by the time of presentation. Endocrine function testing revealed partial hypopituitarism with hypogonadotrophic hypogonadism, secondary adrenal insufficiency, central hypothyroidism, and growth hormone deficiency, but without central diabetes insipidus (CDI) (Table 1). Brain MRI showed a large intra- und suprasellar tumor with severe compression of the optic chiasm. Location and MRI morphology of the tumor were suggestive of a craniopharyngioma. The patient underwent a subtotal resection at the age of 14 years and 9 months. Because of recurrent compression of the optic chiasm, the patient underwent a gross total resection of the craniopharyngioma at the age of 15 years and 10 months. In the following years, the patient gained weight steadily, and quality of life was impacted by marked symptoms of fatigue, daytime sleepiness, and decreased locomotor activity. Treatment with dextroamphetamine was initiated at the age of 17 years and 3 months, 1.5 years after the hypothalamic insult that had likely occurred during the second surgery. Patient 1 had a BMI of 19.3 (z-score -0.3) at the time of craniopharyngioma diagnosis. BMI increased to 29.9 (z-score +1.79 ) prior to initiation of dextroamphetamine. BMI continuously decreased thereafter, reaching 25.3 (z-score +0.4 ) after 4.5 years of uninterrupted treatment. Concurrent with the initiation of dextroamphetamine, daytime sleepiness markedly decreased and activity improved, which allowed the patient to take up a regular employment.

Patient 2

Patient 2 was referred to the department of pediatric oncology following gross total resection of a large suprasellar craniopharyngioma for postoperative treatment. At the time of referral, the girl, aged 14 years and 8 months, suffered from panhypopituitarism with hypogonadotrophic hypogonadism, secondary adrenal insufficiency, central hypothyroidism, growth hormone deficiency, and CDI. In addition, she was affected by adipsia and a severe short-term memory loss. In the 3 months between surgery and dextroamphetamine initiation, the patient experienced rapid, intractable weight gain with a BMI increase from 27.4 (z-score +1.86 ) to 32.0 (z-score +2.59$)$. After initiation of dextroamphetamine treatment, no further increase in BMI z-score occurred until the second year of treatment. Recurrence of weight gain during this phase was attributed to impaired satiety and the recurrence of an uncontrollable drive to eat. Symptoms resolved after stepwise increases of daily dextroamphetamine dose to a maximum of $20 \mathrm{mg}$, leading to a marked reduction of BMI z-score velocity during the third year of treatment (Table 3).

\section{Patient 3}

Patient 3 developed extreme obesity following complete resection of a craniopharyngioma at the age of 21 years and 1 month. She was referred to the endocrine outpatient unit for re-evaluation of her hormonal substitution therapy for panhypopituitarism including CDI (see Table 1), and consequently dextroamphetamine was started 3.5 years after hypothalamic insult. Her BMI at the time of surgery was 27.7 (z-score +1.35 ) and steadily increased to 41.4 (z-score 2.58) by the time treatment was started. During the first 2 years of dextroamphetamine, a significant weight reduction with negative BMI z-score velocity was achieved (Table 3). In the third year of treatment, a moderate weight regain occurred after a patientinitiated reduction of the dextroamphetamine dose from $20 \mathrm{mg} /$ day to $15 \mathrm{mg} /$ day. Dose reduction was motivated by moderate insomnia characterized by delayed sleep initiation, which resolved by implementation of relaxation techniques. 
Table 1. Characteristics of $n=7$ patients with hypothalamic obesity undergoing individual treatment attempts with dextroamphetamine

\begin{tabular}{llllll}
\hline $\begin{array}{l}\text { Patient } \\
\text { ID }\end{array}$ & Sex & $\begin{array}{l}\text { Age at hypotha- } \\
\text { lamic insult, } \\
\text { years }\end{array}$ & Diagnosis & Endocrinopathies & $\begin{array}{c}\text { Time from hypothalamic } \\
\text { insult to start dextroam- } \\
\text { phetamine, years }\end{array}$ \\
\hline 1 & male & 14.8 & craniopharyngioma & HH, CAI, CH, GHD & 2.5 \\
2 & female & 14.7 & craniopharyngioma & HH, CAI, CH, GHD, CDI & 0.2 \\
3 & female & 21.0 & craniopharyngioma & HH, CAI, CH, GHD, CDI & 3.5 \\
4 & female & 11.1 & craniopharyngioma & HH, CAI, CH, GHD, CDI & 5.6 \\
5 & female & 0.5 & meningococcal meningitis & HH, CAI, CH, GHD & 16.5 \\
6 & female & 11.8 & ganglioglioma & CAI, CH & 1.1 \\
7 & male & 12.5 & astrocytoma & HH, CAI, CH, GHD, CDI & 7.2 \\
\hline
\end{tabular}

HH, hypogonadotropic hypogonadism; CAI, central adrenal insufficiency; $\mathrm{CH}$, central hypothyroidism; GHD, growth hormone deficiency; CDI, central diabetes insipidus.

Table 2. Mean daily dextroamphetamine doses during treatment years $1-3$

\begin{tabular}{llll}
\hline $\begin{array}{l}\text { Patient } \\
\text { ID }\end{array}$ & $\begin{array}{l}\text { Mean daily } \\
\text { dextroamphet- } \\
\text { amine dose } \\
\text { 1st year }\end{array}$ & $\begin{array}{l}\text { Mean daily } \\
\text { dextroamphet- } \\
\text { amine dose } \\
\text { 2nd year }\end{array}$ & $\begin{array}{l}\text { Mean daily } \\
\text { dextroamphet- } \\
\text { amine dose } \\
\text { 3rd year }\end{array}$ \\
\hline 1 & $10.4 \mathrm{mg}$ & $13.5 \mathrm{mg}$ & $16.0 \mathrm{mg}$ \\
2 & $13.5 \mathrm{mg}$ & $18.8 \mathrm{mg}$ & $11.7 \mathrm{mg}$ \\
3 & $18.1 \mathrm{mg}$ & $19.0 \mathrm{mg}$ & $16.9 \mathrm{mg}$ \\
4 & $15.0 \mathrm{mg}$ & $20.0 \mathrm{mg}$ & n.a. \\
5 & $7.3 \mathrm{mg}$ & $11.3 \mathrm{mg}$ & $15 \mathrm{mg}$ \\
6 & $9.0 \mathrm{mg}$ & $14.4 \mathrm{mg}$ & n.a. \\
7 & $15.4 \mathrm{mg}$ & n.a. & n.a. \\
\hline
\end{tabular}

Table 3. BMI z-score velocity (see Methods) in $n=7$ patients with hypothalamic obesity undergoing individual treatment attempts with dextroamphetamine before the initiation and during the course of stimulant treatment

\begin{tabular}{|c|c|c|c|c|c|c|c|}
\hline $\begin{array}{l}\text { Patient } \\
\text { ID }\end{array}$ & $\begin{array}{l}\text { BMI z-score } \\
\text { velocity } \\
\text { before insult }\end{array}$ & $\begin{array}{l}\text { BMI z-score at } \\
\text { hypothalamic } \\
\text { insult }\end{array}$ & $\begin{array}{l}\text { BMI z-score at } \\
\text { start dextro- } \\
\text { amphetamine }\end{array}$ & $\begin{array}{l}\text { BMI z-score } \\
\text { velocity } \\
\text { before dextro- } \\
\text { amphetamine }\end{array}$ & $\begin{array}{l}\text { BMI z-score } \\
\text { velocity 1st } \\
\text { year dextro- } \\
\text { amphetamine }\end{array}$ & $\begin{array}{l}\text { BMI z-score } \\
\text { velocity 2nd } \\
\text { year dextro- } \\
\text { amphetamine }\end{array}$ & $\begin{array}{l}\text { BMI z-score } \\
\text { velocity 3rd } \\
\text { year dextro- } \\
\text { amphetamine }\end{array}$ \\
\hline 1 & n.a. & -0.30 & 1.79 & 0.60 & -0.12 & 0.22 & -0.93 \\
\hline 2 & n.a. & 1.86 & 2.59 & n.a. & 0.04 & 0.42 & -0.06 \\
\hline 3 & -0.5 & 1.35 & 2.58 & 0.35 & -0.21 & -0.34 & 0.17 \\
\hline 4 & n.a. & 2.62 & 3.51 & n.a. & -0.32 & -0.21 & n.a. \\
\hline 5 & n.a. & n.a. & 4.20 & n.a. & -0.29 & -0.13 & -0.11 \\
\hline 6 & n.a. & 1.76 & 3.09 & 1.33 & -0.11 & 0.34 & n.a. \\
\hline 7 & n.a. & n.a. & 4.38 & n.a. & -0.22 & n.a. & n.a. \\
\hline \multicolumn{3}{|c|}{ Total group ${ }^{\mathrm{a}}$} & $3.17 \pm 0.93$ & & $-0.18 \pm 0.12$ & $0.05 \pm 0.32$ & $-0.23 \pm 0.48$ \\
\hline \multicolumn{3}{|c|}{ Craniopharyngioma group ${ }^{\mathrm{a}}$} & $2.62 \pm 0.71$ & & $-0.15 \pm 0.15$ & $0.02 \pm 0.36$ & \\
\hline \multicolumn{3}{|c|}{ Others $^{\mathrm{a}}$} & $3.89 \pm 0.7$ & & $-0.21 \pm 0.09$ & & \\
\hline
\end{tabular}

\footnotetext{
${ }^{\text {a }}$ Mean \pm standard deviation.
} 
Denzer et al.: Dextroamphetamine Treatment for Hypothalamic Obesity

\section{Patient 4}

In patient 4 , a craniopharyngioma was diagnosed at the age of 11 years, and gross total resection was performed shortly thereafter. The tumor and neurosurgical procedure led to panhypopituitarism including CDI. At the time of diagnosis, she was already obese with a BMI of 29.8 (z-score +2.62). During 5 years of postsurgical follow-up, BMI increased to 43.2 (z-score +3.51$)$ before dextroamphetamine treatment was initiated. During the first 2 years of stimulant treatment, patient 4 experienced a significant reduction in BMI to 39.3 (z-score +2.84), although by definition still remaining in the extremely obese range. Unfortunately, patient 4 was lost to follow-up during the third year of treatment due to the family's relocation to a distant part of Germany.

Patient 5

This 22-year-old female patient suffered from meningococcal meningitis in infancy. As sequelae of the CNS infection, she was affected by partial hypopituitarism with central adrenal insufficiency, central hypothyroidism, growth hormone deficiency, and hypogonadotropic hypogonadism, but without CDI. Uncontrollable hyperphagia led to extreme obesity in early childhood, resembling the clinical picture of hypothalamic obesity. Multiple interventions to control weight gain and to modify eating behavior were unsuccessful. At initiation of dextroamphetamine treatment, she was 17 years and 4 months old and her BMI was 59.5 (z-score +4.2 ). During the first year of dextroamphetamine treatment, a reduction of BMI to 58.1 (z-score 3.88) could be observed, which represents the patient's first phase of excess weight reduction in more than 15 years. Over the further course of treatment, BMI and BMI z-score stabilized (Table 3).

\section{Patient 6}

Patient 6 underwent surgery for ganglioglioma and tumor-associated hydrocephalus at the age of 11 years and 9 months and was concurrently diagnosed with central adrenal insufficiency and central hypothyroidism, no CDI. During the first year post-surgery, she reported a subjective loss of satiety and experienced massive weight gain with an increase in BMI from 24.6 (z-score +1.76) to 36.4 (z-score + 3.09). She also suffered from low locomotor activity, attention deficit, and impaired short-term memory, which affected academic performance at school. Consequently, she was referred to our institution for evaluation and started on dextroamphetamine treatment. Unfortunately, during the second year of treatment, uncontrolled eating reoccurred and resulted in significant weight gain. Over the same period, increasing psychosocial familial stress significantly impacted daily routine and negatively affected supportive structures, which ultimately prompted a referral to a multidisciplinary, long-term inpatient rehabilitation program at the beginning of year 3 of dextroamphetamine treatment.

\section{Patient 7}

Patient 7 suffered from panhypopituitarism including CDI following incomplete resection of astrocytoma localized between the optic chiasm and the bottom of the third ventricle at the age of 12 years and 6 months. The patient developed onset of refractory obesity in the first year after the neurosurgical intervention. Dextroamphetamine treatment was started at the age of 19 years and 8 months, 7.2 years after the hypothalamic injury, at a BMI of 64.2 (z-score +4.38 ). During the first year of dextroamphetamine treatment, a significant reduction in BMI to 59.5 (z-score 4.27) was achieved, as reflected by the negative BMI z-score velocity (Table 3 ). At the beginning of year 2 of dextroamphetamine treatment, the patient underwent an 18-month inpatient intervention program in a specialized rehabilitation clinic for extremely obese adolescents and young adults, which resulted in weight loss of more than $75 \mathrm{~kg}$. Due to the strong confounding by this intensive multidisciplinary character of the weight loss inter- 
Denzer et al.: Dextroamphetamine Treatment for Hypothalamic Obesity

Fig. 1. Average BMI z-score velocity (see Methods) before and during the first, second, and third year of dextroamphetamine (dex) treatment in $n=7$ patients with hypothalamic obesity. Error bars indicate standard deviations of the mean.

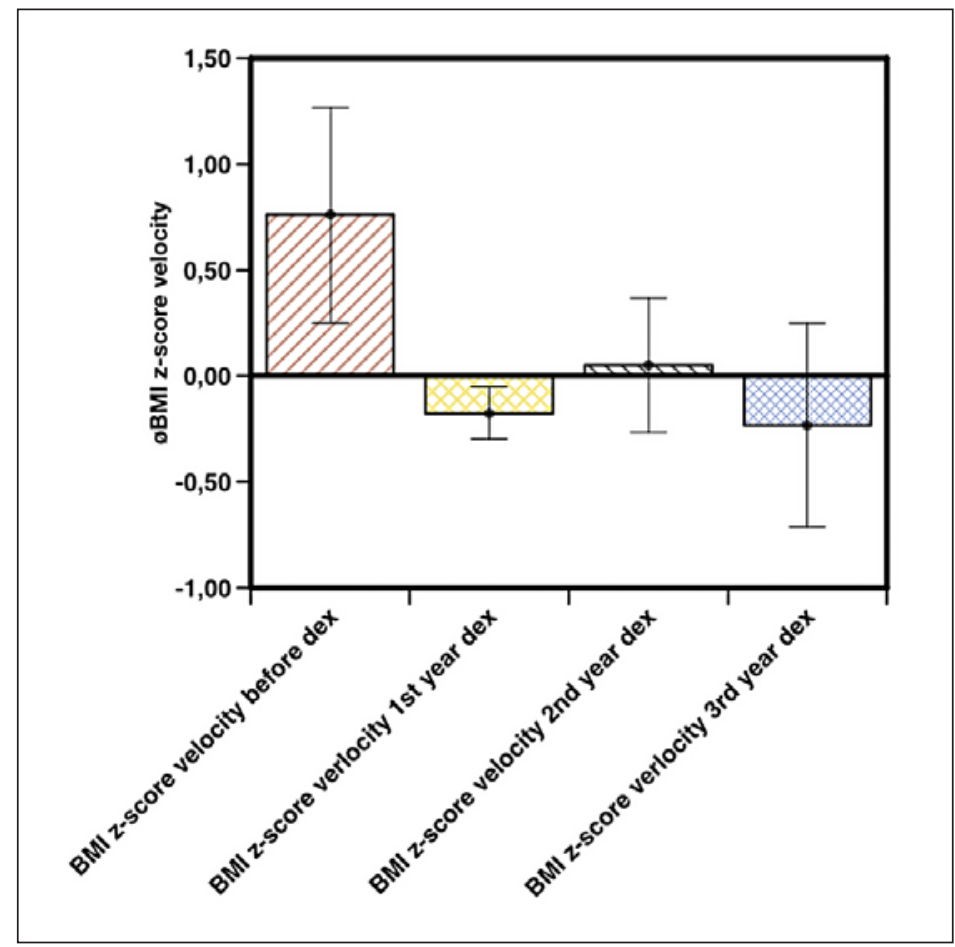

vention, BMI z-scores for this patient are not reported for the second and third year of dextroamphetamine treatment.

\section{Dextroamphetamine Treatment and Dynamics of Change in BMI z-Score}

As detailed in Table 3, BMI z-score of most patients was in the upper normal to moderately obese range at the time of the hypothalamic insult, which coincides with a neurosurgical procedure in all cases except in the patient with meningococcal meningitis. All patients experienced unrelenting increases in BMI z-score following this damaging impact on the hypothalamic region. Unfortunately, we only have complete data sets of anthropometric measurements spanning time periods before the hypothalamic insult and before the start of dextroamphetamine treatment in a minority of patients. In patient 6 , the dramatically high BMI z-score velocity reflects the dynamic weight change typically observed in hypothalamic obesity during the first months after insult, whereas the lower BMI z-score velocities in patients 1 and 3 correspond to the more moderate slope of weight gain during the first years after hypothalamic damage.

All patients were markedly obese at the time of initiation of stimulant treatment, with a mean BMI z-score of $+3.17 \pm 0.93$. Combined results show a notable average reduction of BMI $z$-score during the first year of dextroamphetamine treatment in craniopharyngioma patients as well as in patients with other disease entities (Table 3; Fig. 1,2). Interestingly, the highest BMI z-score velocities were observed in those patients with the shortest intervals between hypothalamic insult and initiation of dextroamphetamine treatment (patients 1, 2, and 6; Table 3), whereas more significant reductions of BMI z-score occurred in patients with longer periods since onset of hypothalamic obesity (patients 3, 4, 5, and 7). In the second year of treatment, a stabilization phase of weight change in terms of average BMI z-score velocity arises (average BMI z-score velocity $+0.05 \pm 0.32$ ). Again, patients with long-standing hypo- 
Denzer et al.: Dextroamphetamine Treatment for Hypothalamic Obesity

Fig. 2. BMI z-score velocity (see Methods) before and during the first, second, and third year of dextroamphetamine (dex) treatment in $n=7$ patients with hypothalamic obesity grouped by diagnosis $(n=4$ patients with craniopharyngioma, $n=3$ patients with astrocytoma, ganglioglioma, meningococcal meningitis).

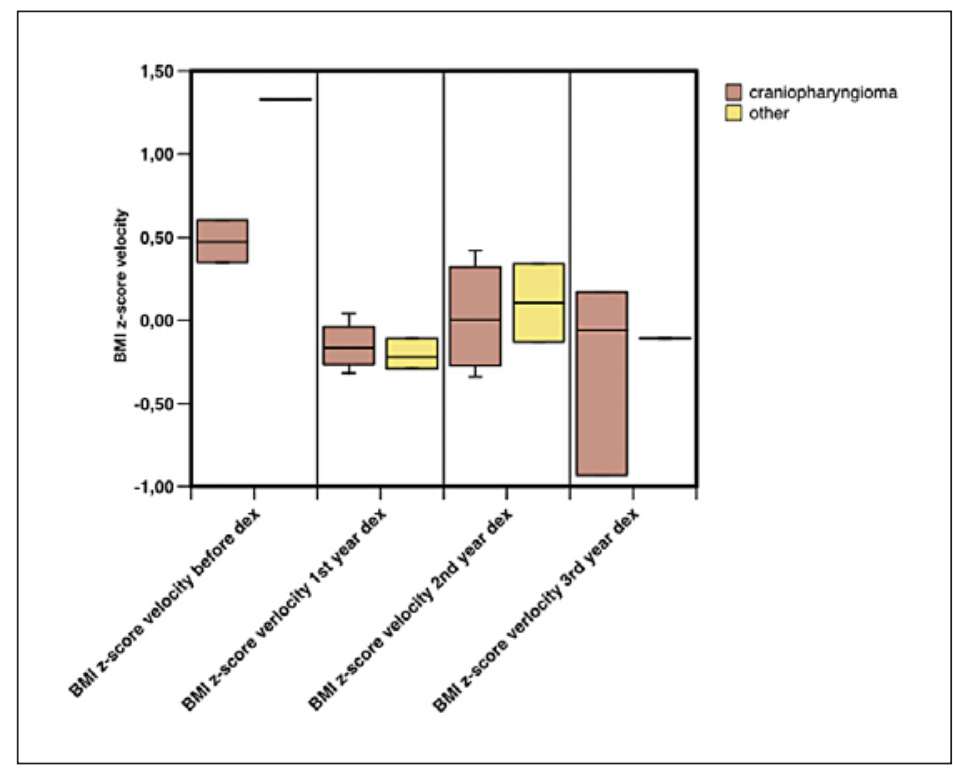

thalamic obesity demonstrate significant decreases in BMI z-score, whereas patients with earlier-phase hypothalamic obesity regain weight (Table 3 ).

In patients with lesions in the sellar region, the manifestation of CDI may serve as a clinical marker for hypothalamic obesity risk. In our cohort, four patients had CDI (patients 2, 3, 4: craniopharyngioma; patient 7: astrocytoma) (Table 1). Time interval from hypothalamic insult to start of dextroamphetamine treatment was heterogenous in both the CDI and the non-CDI group (CDI 0.2-7.2 years, non-CDI 1.1-16.5 years). As shown in Figure 3, BMI z-score velocity fell by a similar rate in both groups during the first year and demonstrated a variable treatment response at the level of the individual patient during the second year of dextroamphetamine.

Over the entire course of dextroamphetamine treatment in all patients, no treatmentrelated side effects or adverse events were recorded.

\section{Discussion}

Hypothalamic obesity is characterized by severe obesity, hyperphagia, decreased satiety, decreased resting energy expenditure and physical activity, as well as marked hyperleptinemia and hyperinsulinemia [3]. Hypothalamic obesity is most often caused by an insult to the hypothalamus due to suprasellar tumors or CNS inflammation as in the present case series. In hypothalamic obesity, impairment of hypothalamic function leads to increased parasympathetic activity and decreased sympathetic activity resulting in autonomic instability [17].

As the hypothalamic (homeostatic) system of energy regulation is closely interrelated with the dopaminergic (non-homeostatic) system, dextroamphetamine may ameliorate or attenuate hypothalamic obesity through both peripheral and central mechanisms. First, through inhibition of reuptake of dopamine, norepinephrine, and serotonin into the presynaptic terminal, as well as stimulation of release of these monoamines from synaptic vesicles by inhibition of the vesicular monoamine transporters (VMAT2) [18, 19], dextroamphetamine is a potent sympathomimetic agent which increases both $\alpha$ - and $\beta$-adrenergic tone. In 
Denzer et al.: Dextroamphetamine Treatment for Hypothalamic Obesity

Fig. 3. BMI z-score velocity (see Methods) before and during the first, second, and third year of dextroamphetamine (dex) treatment in $n=7$ patients with hypothalamic obesity grouped by the extent of hypothalamic involvement as indicated by the presence of central diabetes insipidus (CDI) ( $n=4$ patients with CDI, $n=3$ patients without CDI).

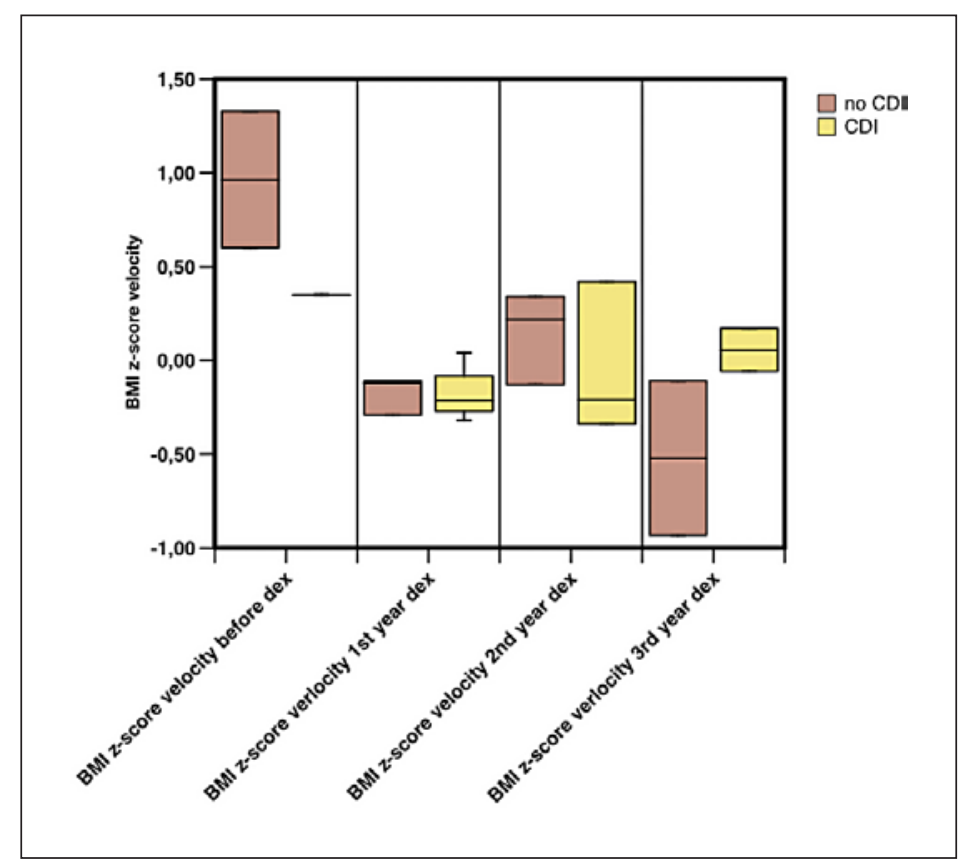

particular, $\beta$-adrenergic function is coupled to peroxisome proliferation activation coactivator- $1 \alpha(\mathrm{PGC}-1 \alpha)$, which is a transcription factor for mitochondrial biogenesis in adipose tissue [20], which can ameliorate excessive weight gain [21]. Second, central anorexigenic effects of dextroamphetamine may be exerted by direct modification of cerebral satiety signaling through stimulation of cocaine-amphetamine regulated transcript (CART), which stimulates a-MSH production to reduce food intake in the ventromedial hypothalamus [22], and reduction of dopamine-mediated food intake in the nucleus accumbens [23]. Indeed, other centrally acting adrenergic psychostimulants, such as methylphenidate, have been utilized for the treatment of hypothalamic obesity in the past, with beneficial effects [24].

In our case series, all but one patient experienced reductions in BMI z-score during the first year of dextroamphetamine treatment. In the patients who reduced overweight, BMI z-score velocity ranged between -0.11 and -0.32 , which represents a remarkable change in body weight development not only in the context of established weight trajectories in hypothalamic obesity, but also compared to common results of lifestyle intervention programs for children and adolescents with "simple" obesity [25].

One female patient who was started on dextroamphetamine less than 3 months after surgery for craniopharyngioma (patient 2) had a stable BMI z-score over the course of the first 12 months on dextroamphetamine (BMI z-score velocity +0.04$)$. Given the excessive rate of weight gain observed in a majority of craniopharyngioma patients with hypothalamic involvement, especially during the first year after diagnosis [6], this finding probably suggests a significant decelerating effect of dextroamphetamine on early rapid weight gain following hypothalamic damage.

Unfortunately, results of the second year of dextroamphetamine treatment are heterogenous with several patients continuing to reduce weight, while others demonstrate significant gains in BMI z-score. Although limited by the small number of available observations, perhaps the potentially beneficial actions of dextroamphetamine treatment on body weight and weight gain depend on the time interval with a primarily stabilizing effect during the first years (patients 1, 2, and 6) and a more pronounced weight-reducing effect during the later years after hypothalamic insult (patients 3, 4, 5, and 7). 
Denzer et al.: Dextroamphetamine Treatment for Hypothalamic Obesity

Regrouping the investigated patients according to underlying diagnosis or employing CDI as a clinical marker for the extent of hypothalamic damage [26] did not reveal differences in treatment response, although results have to be cautiously interpreted due to the observational character of this case series and the overall limited number of available observations.

Results in terms of changes in BMI z-score in our patients treated with dextroamphetamine over a 2- to 3-year period seem to fit within the context of previously published results of similarly small case series [11,12]. Mason et al. [12] reported five patients (age 6.0-9.8 years) started on dextroamphetamine treatment for unrelenting weight gain and attention deficit disorder 7-14 months following neurosurgical intervention for craniopharyngioma. During 24 months of dextroamphetamine treatment with a maximum dose of $20 \mathrm{mg} /$ day, absolute BMIs of participants stabilized and weight velocity (calculated as weight change in $\mathrm{kg} / \mathrm{month}$ ) dropped considerably compared to pretreatment values. In addition, significant improvements in hyperactivity scores as measured by standardized rating scales were reported by parents and teachers. Similarly, Ismail et al. [11] report on weight change and wakefulness in 12 patients with hypothalamic lesions (craniopharyngioma $n=9$, astrocytoma $n=2$, glioma $n=1$ ) causing hypothalamic obesity treated with lower-dose dextroamphetamine (5 mg p.o. b.i.d.) over the course of 7-45 months. After a median treatment duration of 13 months in boys and 15 months in girls, in 10 of 12 patients either weight loss or stabilization was achieved. The observed median loss in weight z-score in the study population amounted to -0.7 in males and -0.44 in females.

Although comparability between patients and case series is strictly limited due to methodological discrepancies and differences in patients' characteristics, dextroamphetamine treatment resulted in weight stabilization or even moderate to significant weight loss in all three patient groups (total $n=24$ ). Furthermore, Mason et al. [12] and Ismail et al. [11] report other beneficial effects of stimulant treatment such as increased wakefulness, attention, and activity. These findings are consistent with subjective reports of our patients, although in our experience, potential positive effects on these traits seem to be variable and subject to confounding by significant disabilities and comorbidities (e.g., obstructive sleep apnea, impairments in psychomotor function). In our case series, dextroamphetamine treatment was well tolerated and no adverse side effects were recorded as in both previously published studies. Although medication-related side effects may be subject to significant underreporting, reassuringly, no patient asked for cessation or stopped dextroamphetamine treatment over the entire follow-up period. Due to the observational character of the individual treatment courses presented here, no objective measures of patients' compliance with regard to regular drug use were available. Therefore, individual responses to dextroamphetamine might have been impacted by low or variable compliance.

In conclusion, this case series underscores the potential role of dextroamphetamine treatment in hypothalamic obesity in children and adolescents. Dextroamphetamine treatment may lead to an attenuation of excessive weight gain during the early phase after hypothalamic insult and furthermore may facilitate more pronounced weight loss during longer-term follow-up. Larger treatment trials are needed to further elucidate the efficacy of dextroamphetamine treatment and to determine optimal timepoints for initiation of therapy in patients with hypothalamic obesity. Unfortunately, due to its potential for abuse, dextroamphetamine is not available in some countries, e.g. UK and India. Furthermore, dextroamphetamine, or more generally adrenergic agonist treatment, should be considered as a potential component in a multimodal treatment strategy incorporating differential pharmacologic (e.g., octreotide, metformin, leptin-sensitizing drugs, optimized hormone replacement) and individualized supportive and motivational psychotherapeutic approaches for patients affected by hypothalamic obesity. 
Denzer et al.: Dextroamphetamine Treatment for Hypothalamic Obesity

\section{Statement of Ethics}

The present case series is of mere observational character, data was collected retrospectively from patient files. Treatment with dextroamphetamine was initiated as individual treatment attempts in the context of routine patient care, which do not require individual approval by the institutional review board. There was no study protocol and no clinical or laboratory investigations or other monitoring exceeding common medical practice in this patient group. Therefore, the present case series data does neither qualify as clinical trial of a pharmaceutical product nor as any form of post-authorisation study ( $\$ 4$ Abs. 23 Satz 1 and Satz 3 AMG (German Pharmaceuticals Act)).

\section{Disclosure Statement}

No potential conflicts of interest relevant to this article were reported.

\section{Author Contributions}

C.D. researched data and wrote the manuscript. F.D. researched data and reviewed and edited the manuscript. B.S.L. contributed to discussion and reviewed and edited the manuscript. H.V. reviewed and edited the manuscript. R.H.L. contributed to discussion and reviewed and edited the manuscript. M.W. oversaw individual treatment attempts with dextroamphetamine and reviewed and edited the manuscript.

\section{References}

1 Bereket A, Kiess W, Lustig RH, Muller HL, Goldstone AP, Weiss R, et al. Hypothalamic obesity in children. Obes Rev. 2012 Sep;13(9):780-98.

2 Lustig RH. Hypothalamic obesity: causes, consequences, treatment. Pediatr Endocrinol Rev. 2008 Dec;6(2): 220-7.

3 Lustig RH. Hypothalamic obesity after craniopharyngioma: mechanisms, diagnosis, and treatment. Front Endocrinol (Lausanne). 2011 Nov;2:60.

4 Haliloglu B, Bereket A. Hypothalamic obesity in children: pathophysiology to clinical management. J Pediatr Endocrinol Metab. 2015 May;28(5-6):503-13.

5 Müller HL. Childhood craniopharyngioma - current concepts in diagnosis, therapy and follow-up. Nat Rev Endocrinol. 2010 Nov;6(11):609-18.

6 Müller HL, Emser A, Faldum A, Bruhnken G, Etavard-Gorris N, Gebhardt U, et al. Longitudinal study on growth and body mass index before and after diagnosis of childhood craniopharyngioma. J Clin Endocrinol Metab. 2004 Jul;89(7):3298-305.

7 Roth CL. Hypothalamic Obesity in Craniopharyngioma Patients: Disturbed Energy Homeostasis Related to Extent of Hypothalamic Damage and Its Implication for Obesity Intervention. J Clin Med. 2015 Sep;4(9):177497.

8 Roth CL, Hunneman DH, Gebhardt U, Stoffel-Wagner B, Reinehr T, Müller HL. Reduced sympathetic metabolites in urine of obese patients with craniopharyngioma. Pediatr Res. 2007 Apr;61(4):496-501.

9 Faraone SV, Biederman J, Morley CP, Spencer TJ. Effect of stimulants on height and weight: a review of the literature. J Am Acad Child Adolesc Psychiatry. 2008 Sep;47(9):994-1009.

10 Haslam D. Weight management in obesity - past and present. Int J Clin Pract. 2016 Mar;70(3):206-17.

11 Ismail D, O'Connell MA, Zacharin MR. Dexamphetamine use for management of obesity and hypersomnolence following hypothalamic injury. J Pediatr Endocrinol Metab. 2006 Feb;19(2):129-34.

12 Mason PW, Krawiecki N, Meacham LR. The use of dextroamphetamine to treat obesity and hyperphagia in children treated for craniopharyngioma. Arch Pediatr Adolesc Med. 2002 Sep;156(9):887-92.

13 Kromeyer-Hauschild K, Moss A, Wabitsch M. Referenzwerte für den Body-Mass-Index für Kinder, Jugendliche und Erwachsene in Deutschland. Adipositas - Ursachen, Folgen. Therapie. 2015;9:123-7.

14 Kromeyer-Hauschild K, Wabitsch M, Geller F, Ziegler A, Geiß HC, Hesse V, et al. Perzentilen für den BodymassIndex für das Kindes- und Jugendalter unter Heranziehung verschiedener deutscher Stichproben. Monatsschr Kinderheilkd. 2001;149(8):807-18.

15 Cole TJ. The LMS method for constructing normalized growth standards. Eur J Clin Nutr. 1990 Jan;44(1): 45-60.

16 Tanner JM. The assessment of growth and development in children. Arch Dis Child. 1952 Feb;27(131):10-33.

17 Cohen M, Syme C, McCrindle BW, Hamilton J. Autonomic nervous system balance in children and adolescents with craniopharyngioma and hypothalamic obesity. Eur J Endocrinol. 2013 May;168(6):845-52. 
18 Seiden LS, Sabol KE, Ricaurte GA. Amphetamine: effects on catecholamine systems and behavior. Annu Rev Pharmacol Toxicol. 1993;33(1):639-77.

19 Wilens TE. Effects of methylphenidate on the catecholaminergic system in attention-deficit/hyperactivity disorder. J Clin Psychopharmacol. 2008 Jun;28(3 Suppl 2):S46-53.

20 Sutherland LN, Bomhof MR, Capozzi LC, Basaraba SA, Wright DC. Exercise and adrenaline increase PGC1 \{alpha\} mRNA expression in rat adipose tissue. J Physiol. 2009 Apr;587(Pt 7):1607-17.

21 Leone TC, Lehman JJ, Finck BN, Schaeffer PJ, Wende AR, Boudina S, et al. PGC-1alpha deficiency causes multisystem energy metabolic derangements: muscle dysfunction, abnormal weight control and hepatic steatosis. PLoS Biol. 2005 Apr;3(4):e101.

22 Lau J, Farzi A, Qi Y, Heilbronn R, Mietzsch M, Shi YC, et al. CART neurons in the arcuate nucleus and lateral hypothalamic area exert differential controls on energy homeostasis. Mol Metab. 2018 Jan;7:102-118.

23 Yang SC, Shieh KR, Li HY. Cocaine- and amphetamine-regulated transcript in the nucleus accumbens participates in the regulation of feeding behavior in rats. Neuroscience. 2005;133(3):841-51.

24 Elfers CT, Roth CL. Effects of methylphenidate on weight gain and food intake in hypothalamic obesity. Front Endocrinol (Lausanne). 2011 Dec;2:78.

25 Denzer C, Reithofer E, Wabitsch M, Widhalm K. The outcome of childhood obesity management depends highly upon patient compliance. Eur J Pediatr. 2004 Feb;163(2):99-104.

26 Roth CL, Eslamy H, Werny D, Elfers C, Shaffer ML, Pihoker C, et al. Semiquantitative analysis of hypothalamic damage on MRI predicts risk for hypothalamic obesity. Obesity (Silver Spring). 2015 Jun;23(6):1226-33. 